\title{
THE ADOPTION OF THE INTERNET OF THINGS FOR SMART AgRICULTURE IN ZIMBABWE
}

\author{
Tsitsi Zengeya ${ }^{1}$, Paul Sambo ${ }^{1}$ and Nyasha Mabika ${ }^{2}$ \\ ${ }^{1}$ Great Zimbabwe University, Department of Mathematics \\ and Computer Science \\ ${ }^{2}$ Great 2Zimbabwe University, Department of Livestock, Wildlife and Fisheries
}

\begin{abstract}
Zimbabwe has faced severe droughts, resulting in low agricultural outputs. This has threatened food and nutrition security in community sections, especially in areas with low annual rainfall. There is a growing need to maximize water usage, monitor the environment and nutrients, and temperatures by the adaptation of smart agriculture. This research explored the use of the Internet of Things (IoT) for smart agriculture in Zimbabwe to improve food production. The mixed methodology was used to gather data through interviews from 50 purposively sampled A2 farmers in the five agricultural regions of Zimbabwe and was supported by the use of the Internet. The findings reveal that some farmers have adopted IoT in Zimbabwe, others are still to adopt such technology and some are not aware of the technology. IoT's benefits to Zimbabwean farmers are immense in that it improves food security, water preservation, and farm management. However, for most farmers to benefit from IoT, more awareness campaigns should be carried out and mobile and fixed Internet connectivity improved in some of the areas.
\end{abstract}

\section{KEYWORDS}

Internet of Things, Adoption, Smart Agriculture, Activity Theory, Covid-19.

\section{INTRODUCTION}

In Zimbabwe agriculture forms the backbone of the economy by contributing approximately $17 \%$ of the Gross Domestic Product (GDP). Farming activities generate an income for about $60-70 \%$ of the population [1]. The Zimbabwean agricultural sector is composed of crop production, animal production, and forestry (tree plantations) [2]. This sector has seen a decline in food production, deforestation resulting in the country importing major food items such as maize, wheat, and soya beans. The introduction of technology in agriculture has boosted food production in some of the developed countries especially in the United States of America (USA) and other developing countries [3]. While most African countries are still facing difficulties in food security, later alone the adoption of technology in the agricultural sector remains a challenge.

In1999 to 2000, the Zimbabwean agricultural sector did undergo agrarian reforms to equitably share land which had been caused by colonial imbalance. Most of the farms were subdivided so that more farmers would be accommodated. The farms were previously described as communal, resettlement, small-scale commercial, and large-scale commercial farms. During the agrarian reforms, the farms were modelled along with A1 and A2 models. A1 model farms are divided into small plots where a number of villagers are apportioned 5 hectares of arable land with 
communal grazing. A2 model comprises of autonomous farms with commercial activities. The farming activities for both A1 and A2 models are based on the agro-ecological region[4].

Five agro-ecological regions in Zimbabwe are classified as natural regions. These regions are categorized according to the amount of rainfall, soil quality, vegetation, climatic conditions among other factors. Zimbabwe's rainfall pattern ranges from 550 to 900 millimeters across the five regions. Most of the Zimbabwean farmers rely on rainfall for crop farming and some of the A1 and A2 farms largely rely on irrigation [5]. Zimbabwe has experienced food shortages due to droughts, storms, and floods. This has forced the country to import food for the past decade [6]. There is a growing need for Zimbabwean farmers to utilize technology to improve food security.

\section{LiteratURE REVIEW}

Internet of Things is defined as a network of interconnected devicessuch as sensors and communication networksconnected through the internet to transfer information without human intervention [7]. IoT has managed to change the traditional method of farming, by aiding farmers with the use of technology. This has transformed the agricultural sector from precision farming into smart farming [8]. A farmer can monitor their field with the use of sensors like dielectric soil moisture sensors. The sensors give an opportunity for a farmer to plan watering times and areas that need to be irrigated the most. Sensors can also be used to monitor and alert the farmer on the movement of pests in the field. IoT allows farmers to remotely control farm activities, processing, and logistic operations by the use of sensors and actuators, e.g. it allows for accuracy in the application of pesticides and fertilizers or robots for automatic weeding. IoT can be used to monitor food quality during transportation by remotely accessing and controlling the geographic location and conditions of shipments and products.

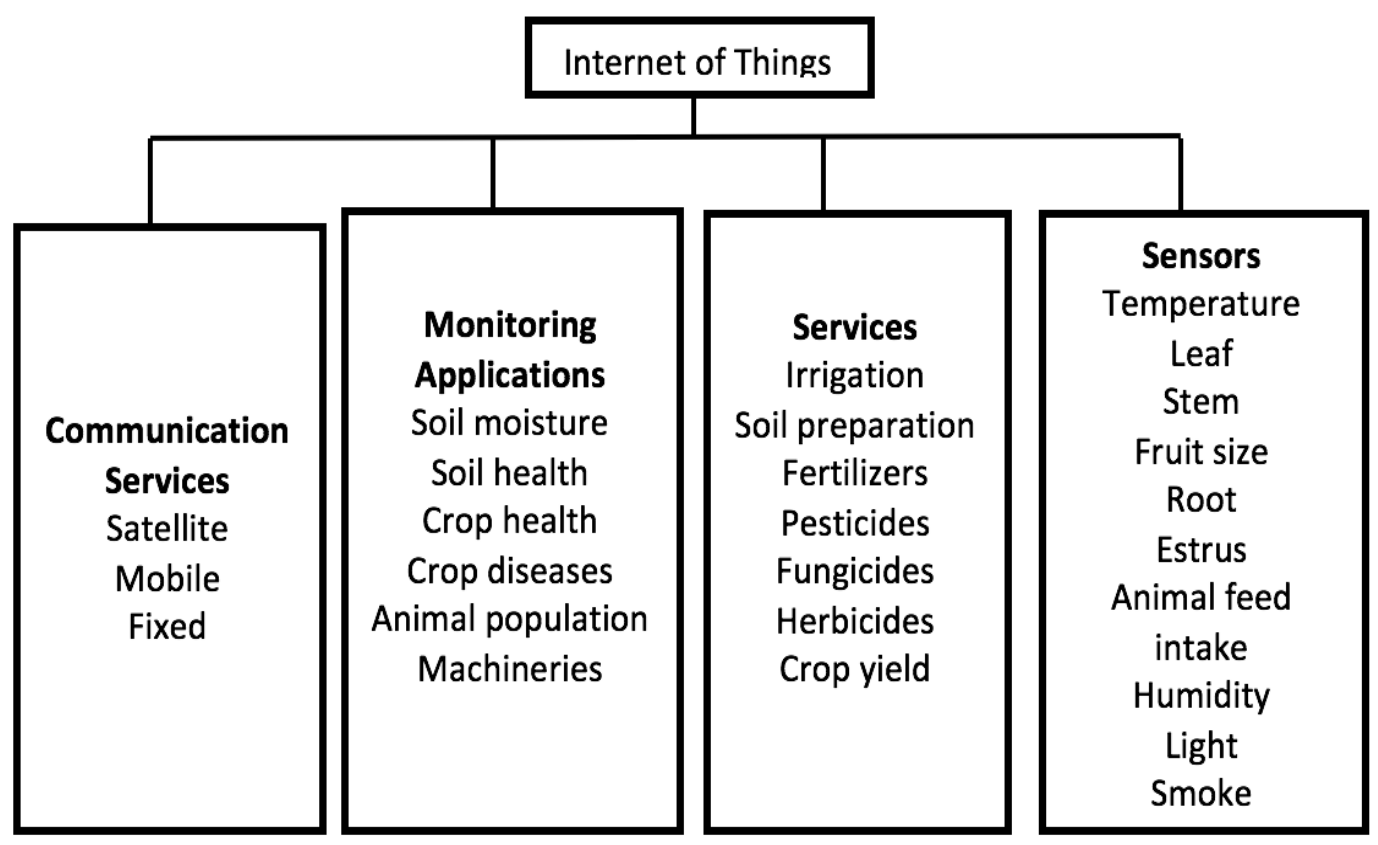

Figure 1. Illustration of Internet of Things in agriculture

Figure 1 illustrates the application of the Internet of things in agriculture which is composed of four elements, communication services, monitoring applications, services, and sensors. Communication services include network services for Internet data that can be offered through 
satellite, mobile fixed networks [9]. Data transmission of IoT devices varies and can be supported with $2 \mathrm{G}-5 \mathrm{G}$ cellular networks. Internet-linked devices enable farmers to collect and exchange data without human involvement. The monitoring applications can be used to monitor soil moisture, soil health, crop health, crop diseases, and animal population [10]. Machinery such as combine harvesters, tractors, irrigation equipment, and drones can be fitted with sensors [11], for example, Hello Tractor developed a low-cost monitoring device that can be used to monitor the condition of the tractor [12]. IoT can be applied through an agricultural drone which is a relatively inexpensive device fitted with a mechanism that provides farmers with information about the status of the crops which can result in an increase in yields and reduce crop damage.The drone can also be used to track and monitor the movement of animals and check if there is any danger being paused in their area [13]. IoT can also be used with irrigation equipment where water usage can be monitored. The services include the detection of soil nutrients and the amount of fertilizer required. The services for IoT in agriculture vary from crop yield to, detection of pests and herbs affecting the growth of the crop. Sensor devices play a pivotal role in the collection of data about the status of the land, crop, or animal, for example, the devices can be used to determine fruit size, moisture, or nutrient content [14].

With the adoption of IoT, farmers will be able to control the internal processes and thereby decrease production risks. The availability of data allows farmers to foresee the output of production and allows for better planning especially crop management and product distribution. With enhanced control over overproduction, waste levels can be reduced and costs can be more effectively managed. Knowledge about any anomalies or challenges in the rate of crop growth or the health of livestock allows farmers to mitigate the risk of diminished yield or even crop failure [15],.

To implement an effective and successful IoT solution in agriculture three factors should be considered:i) allowing for real-time collection and presentation of data, ii)providing a solution that is low-powered, easy to install, and cost-effective and iii) provide a solution that can be remotely accessed globally and not restricted to the operator or local networks [16]. Farmers are more worried about farm management and increase in production, rather than being bogged down about technical and costs issues of IoT.

Current agricultural trends have seen the adoption of novel strategies of crop production such as greenhouses, hydroponics, vertical farming, and phenotyping to increase crop yield [14]. Crop production in greenhouses is done in a controlled environment which allows for seasoned and unseasoned crops to be grown anywhere at any time. Wireless communication, mobile devices, and other Internet devices are used in the greenhouse to monitor humidity, temperature, light, and pressure. Hydroponics allows farmers to grow seasonal and unseasonal crops in water under controlled conditions without a soil medium and the nutrients are applied through the irrigation system. Wireless devices connected through the Internet are used to monitor the water level, nutrients, and fertilizers used for crop production. Vertical farming allows farmers to grow crops in a controlled environment on a small piece of land. This type of farming is commonly used in Japan [17]. The use of IoT in vertical farming permits the control of moisture and groundwater using computers or cellular devices such as tablets and smartphones. Phenotyping "is an advanced genetic engineering technique and biotechnology which correlates the genetic sequences of crops for agronomical and physiological aspects" [18]. In this approach, IoT is used to determine and analyze the characteristics of genetic engineering and biotechnology of the crops [7].

IoT is also being used to improve the sustenance of food production in aquaculture. Aquaculture is an agricultural activity where farmers focus on producing fish, water plants, and diverse oceanic organisms [19]. Devices can be used to monitor the water, oxygen and nutrients levels 
and transmit this data through the Internet. Aquaponics is a sustainable agriculture in a symbiotic environment by combining aquaculture and hydroponics [20]. The water system should flow on the planting medium periodically to ensure the plants get the nutrients, while the water can be filtered properly by the medium.

IoT can be used with cloud computing which resolves some of the limitations of the devices and sensors, by providing storage solutions and computing power for analysis. Cloud computing also offers farmers an opportunity to obtain valuable information about markets, especially seeds, fertilizers, equipment, and farming methods. Cloud computing can also facilitate the use of Big Data analytical tools for farmers [21] [22].

Most farmers in Zimbabwe spend their time physically monitoring and understanding farming activities while modern agricultural activities require better farming management techniques through the adoption of technology. In developed countries, farmers have adopted intelligent farming systems to improve agricultural activities [23]. Zimbabwe has been affected by severe droughts in the past two decades which has resulted in lower food production. Does the adoption of technology (IoT) efficiently and effectively help improve agricultural production in Zimbabwe? This paper investigated how the adoption of IoT for smart agriculture in Zimbabwe will help improve food security.

The theoretical framework of this research is based on the activity theory which seeks to describe the socio-technical activities in agriculture. The activity theoryhelped the researchers in understanding the processes, actors involved in farming activities, and how the adoption of IoT will benefitZimbabwean farmers.

\section{RESEARCH METHODOLOGY}

The mixed methodology was used in this research involving the collection of qualitative data throughinterviews and documentation. 50 farmers from A2 model farms in the five agroecological regions were interviewed online due to the Covid- 19 restrictions. The Covid -19 restrictions in Zimbabwe limited the movement of people to curb the transmission of the disease. Data was also gathered from the Internet about the adoption of IoT in the Zimbabwean agricultural sector. Farmers who had access to the Internet and Social media platforms were identified through purposive sampling. The six tenets of activity theory: the objective of the IoT, the actors involved, the agricultural community, the technology (IoT), the division of activities among the actors in the agricultural system, and regulations governing the use of IoT in Zimbabwe were used as guidelines for the research. Data was then analyzed and categorized into three clusters, i) farmers who had adopted IoT, ii) farmers knowledgeable about IoTbut had not adopted such technology, and iii) those who were not aware of such technology.

\section{FINDINGS}

From the interviews held online, three clusters emerged, farmers who had adopted IoT, farmers knowledgeable about IoT but still to adopt such technology, and others who were not aware of such technology.

\subsection{Farmers Who Have Adopted IoT in Agriculture}

During the interviews, some of the farmers revealed that they had adopted IoT and the benefits were quite enormous. The farms that had adopted IoT, were able to monitor soil nutrients, moisture, water usage, temperature, humidity, light, weed, and pests. Some of the farms had 
sensors in their greenhouses to monitor the environmental parameters for example the sensors were able to monitor temperatures, humidity, soil nutrients, and light. In one of the farms, the farmer installed global tracking devices on some of the bull cattle. This assistedthe farmer in animal management i.e. ability to locate the animals if they had been lost or stolen.

The following benefits were highlighted on the farms that were adopting IoT in Zimbabwe: enhanced decision making, savings in electricity, preservation of water, better yields, and reduced labour. The farmers indicated that they were able to monitor their fields or animals remotely and could make faster decisions especially if they had challenges on the farm. The farmers also stated that electricity was saved due to constant monitoring of the moisture content of the soil rather than physically checking the wetness of the ground. The farmers also revealed that water usage was reduced because only areas that needed to be irrigated would get the required amount of water. There was an improvement in the yields as farmers were able to monitor the growth of their plants especially the soil nutrients and other adverse weather conditions. Labour costs were also reduced as the farmers did not have to send someone to the field to physically check the temperatures, moisture, or nutritious content. This data would be remotely transmitted to the farmer.

\subsection{Farmers Knowledgeable about IoT}

During the interviews, some farmers were knowledgeable about IoT, but are still adopting the technology. The reasons that were given by the farmers for not adopting such technology were cost, lack of proper infrastructure, poor internet connectivity, and the requisite skill to adopt such systems. The farmers stated that adopting such technology using the existing mobile or fixed networks had challenges during access, uploading, and downloading data as services were poor and not accessible in some other areas. The other alternative which is satellite services were said to be costly in Zimbabwe.

\subsection{Farmers still to adopt IoT in agriculture}

The majority of the farmers interviewed were not aware of the IoT technology and its benefits and it was their first time to be introduced to such technology. Some of the farmers who did not know about the existence of such technology revealed that they were eager to embrace this innovative technology. But, some of the farmers, although made aware of IoT in agriculture through this interview said they would not adopt such technology.

\section{DiscuSSION AND ANALYSIS}

In some of the farms in Zimbabwe, IoT has changed the traditional methods of farming to Smart Farming. Farming activities have formed a smart web of interoperable farm objects. With the adoption of IoT, farm management is integrated by real-time sensing and monitoring, smart analysis and planning, and smart control of all relevant farm processes [24].

IoT can be adopted by monitoring the seeds, plantation, harvesting, and quality of the products during the whole cycle of crop production. Many benefits come with the adoption of IoT by Zimbabwean farmers such as remote monitoring of farming activities and enhancing the decision-making process as evidenced in section 4.1. Although the benefits of IoT in agriculture are enormous, some farmers are not knowledgeable about such technology in Zimbabwe. This is supported by [25] who state that the lower levels of education in technologies in developing countries affect the technological acceptance by farmers as they are more comfortable with traditional methods of farming as compared to the modern techniques. [25] further alludes that 
most farmers pride themselves in crop and animal production, the terrain and soil quality of their land, and are reluctant to adopt high-tech monitoring technology.

A developing country like South Africa is implementing IoT in agriculture examples are in the wine industry which monitors the whole cycle of growing grapes up to the level of wine production. IoT is also used in other crop production such as potatoes, maize production water, and livestock monitoring [26].

Real-time communication plays an important role in the adoption of technology in advanced agriculture. Communication enhances faster decision making thereby enabling farmers to manage farm activities effectively [27]. Network service providers should provide services that will benefit farmers in the adoption of IoT. The advancement of IoT requires more bandwidth and the cost of data from the network service providers should be affordable to farmers in Zimbabwe. However, when approached effectively, the adoption of IoT and more broadly information and communication technology in developing countries like Zimbabwe can contribute towards food security.

By using smart agriculture technology, Zimbabwean farmers will gain better control of farming activities such as the rearing of livestock and growing crops, bringing about massive efficiencies of scale, cutting costs, and helping save scarce resources such as water.As Zimbabwe is often affected by droughts, IoT will allow the country to preserve water. With the adoption of IoT, Zimbabwe will be able to provide smart solutions in agriculture.

\section{Conclusions}

The use of IoT has immense benefits to Zimbabwean food security as farmers will be able to make faster decisions thereby boosting agricultural productivity. IoT will enable framers to monitor soil nutrients, environmental parameters, water usage and enabling farmers to be well informed about agricultural activities in their fields regardless of geographic area.

However, it is important to note some of the farmers are not aware of IoT and some of the agricultural regions have poor Internetconnectivity making it difficult to adopt such technology. It is recommended that awareness campaigns should be conducted for the adaptation of IoT by farmers in Zimbabwe. In order for Zimbabwean farmers to also benefit from the adoption of IoT, communication infrastructure coverage and speed of the Internet should be improved.

\section{ACKNOWLEDGEMENTS}

This paper is inspired by the research that has been done about IoT in the Zimbabwean agriculture sector.

\section{REFERENCES}

[1] FAO, "Zimbabwe at a glance: Food and Agriculture Organization of the United Nations," FAO Zimbabwe. 2018.

[2] Ministry of Agriculture, "Comprehensive Agricultural Policy Framework," 2012.

[3] OECD, "Adoption of Technologies for Sustainable Farming Systems," in Wageningen Workshop Proceedings, 2001, p. 147.

[4] S. Moyo, "Review of African Political Economy," JSTOR, vol. 38, no. 128, pp. 257-276, 2011.

[5] FAO, Fertilizer use by crop in Zimbabwe. 2004.

[6] K. Nyikahadzoi, "Drought Hazard Risk and Humanitarian Impact Analysis and Inventorisation of Forecast Models in Zimbabwe," 2021.

[7] N. Khan, R. L. Ray, G. R. Sargani, M. Ihtisham, M. Khayyam, and S. Ismail, "Current progress and 
future prospects of agriculture technology: Gateway to sustainable agriculture," MDPI, vol. 13, no. 9 , pp. 1-31, 2021.

[8] V. Saiz-Rubio and F. Rovira-Más, "From smart farming towards agriculture 5.0: A review on crop data management," MDPI, vol. 10, no. 2, 2020.

[9] J. E. Sierra, B. Medina, and J. C. Vesga, "Management system in intelligent agriculture based on Internet of Things," Espacios, vol. 39, no. 8, 2018.

[10] H. M. Jawad, R. Nordin, S. K. Gharghan, A. M. Jawad, and M. Ismail, "Energy-efficient wireless sensor networks for precision agriculture: A review," MDPI, vol. 17, no. 8, 2017.

[11] FAO, "Agriculture 4.0: Start Agricultural robotics and automated equipment for sustainable crop production," 2020.

[12] Hello Tractor, "Hello Tractor, Break Ground, Drive Change."

[13] R. Giacomo and G. David, E-Argriculture in action: Drones for agriculture. 2018.

[14] M. Ayaz, M. Ammad-Uddin, Z. Sharif, A. Mansour, and E. H. M. Aggoune, "Internet-of-Things (IoT)-based smart agriculture: Toward making the fields talk," IEEE Access, vol. 7, pp. 129551129583, 2019.

[15] R. A. Acharige, M. N. Halgamuge, H. A. H. S. Wirasagoda, and A. Syed, "Adoption of the Internet of Things ( IoT ) in Agriculture and Smart Farming towards Urban Greening : A Review," Int. J. Adv. Comput. Sci. Appl., no. April, pp. 10-28, 2019.

[16] C. Verdouw, S. Wolfert, and B. Tekinerdogan, "Internet of things in agriculture," CAB Rev. Perspect. Agric. Vet. Sci. Nutr. Nat. Resour., vol. 11, no. October 2017, 2016.

[17] K. Benke and B. Tomkins, "Future food-production systems: vertical farming and controlledenvironment agriculture," Sustain. Sci. Pract. Policy, vol. 13, no. 1, pp. 13-26, Jan. 2017.

[18] N. Khan, R. L. Ray, G. R. Sargani, M. Ihtisham, M. Khayyam, and S. Ismail, "Current progress and future prospects of agriculture technology: Gateway to sustainable agriculture," Sustain., vol. 13, no. 9, 2021.

[19] U. Acar et al., "Designing An IoT cloud solution for aquaculture," Glob. IoT Summit, GIoTS 2019 Proc., no. June, 2019.

[20] C. Li et al., "Prospect of aquaponics for the sustainable development of food production in urban," Chem. Eng. Trans., vol. 63, no. August, pp. 475-480, 2018.

[21] G. Vitali, M. Francia, and M. Golfarelli, "Crop Management with the IoT: An Interdisciplinary Survey," MDPI, pp. 1-18, 2021.

[22] S. Nandhini, S. Bhrathi, D. D. Goud, and K. P. Krishna, "Smart Agriculture IOT with Cloud Computing, Fog Computing and Edge Computing," Int. J. Eng. Adv. Technol., vol. 9, no. 2, pp. 3578-3582, 2019.

[23] A. Walter, R. Finger, R. Huber, and N. Buchmann, "Opinion: Smart farming is key to developing sustainable agriculture," in Proceedings of the National Academy of Sciences, 2017, vol. 114, no. 24, pp. 6148-6150.

[24] N. Dlodlo and J. Kalezhi, "The internet of things in agriculture for sustainable rural development," Proc. 2015 Int. Conf. Emerg. Trends Networks Comput. Commun. ETNCC 2015, pp. 13-18, 2015.

[25] E. Botha, R. Malekian, and O. E. Ijiga, "IoT in Agriculture: Enhanced Throughput in South African Farming Applications," 2019 IEEE 2nd Wirel. Africa Conf. WAC 2019 - Proc., no. September, pp. 15, 2019.

[26] P. Aguera, N. Berglund, T. Chinembiri, A. Comninos, A. Gillwald, and N. Govan-Vassen, "Paving the way towards digitalising agriculture in South Africa," no. June, pp. 1-42, 2020.

[27] I. Lee and K. Lee, "The Internet of Things (IoT): Applications, investments, and challenges for enterprises," Sci. Direct, vol. 58, no. 4, pp. 431-440, 2015. 


\section{AUTHORS}

Tsitsi Zengeya is a Computer Science lecturer at Great Zimbabwe University. She holds an Msc degree in Computer Science. Areas of research interest are: Artificial Intelligence, Data Science \& Ontologies.

Dr. Paul Sambo is a lecturer at Great Zimbabwe University's Computer Science department. He has wealth of experience in the Information Communication Technology industry. He holds a PhD in Information Systems and his research areas are: Information systems, Data Communication, Artificial Intelligence and Software Engineering.
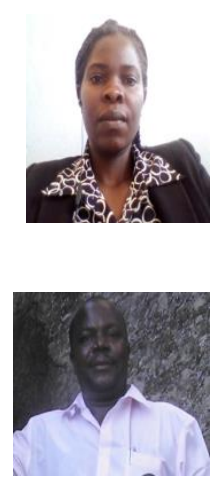

Nyasha Mabika is a lecturer at Great Zimbabwe University, Department of Livestock, Wildlife and Fisheries. He has vast experience in biological techniques and fishery sciences. He is a $\mathrm{PhD}$ holder with an interest in fish health and bio monitoring.

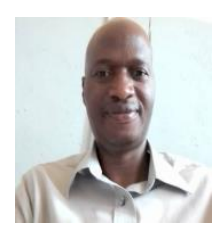

(C) 2021 By AIRCC Publishing Corporation. This article is published under the Creative Commons Attribution (CC BY) license. 